\title{
Expression of Rice (Oryza sativa L.) Genes Involved in High-Affinity Nitrate Transport during the Period of Nitrate Induction
}

\author{
Ryoichi Araki ${ }^{1)}$ and Hiroshi Hasegawa ${ }^{* 1,2)}$ \\ 1) Graduate School of Environmental Science, The University of Shiga Prefecture, 2500 Hassaka, Hikone, Shiga 522-8533, Japan \\ 2) School of Environmental Science, The University of Shiga Prefecture, 2500 Hassaka, Hikone, Shiga 522-8533, Japan
}

\begin{abstract}
When breeding crops that utilize nitrogen efficiently, it is important to reveal the regulation of nitrate uptake at the molecular level. We focused on the expression of two nitrate uptake-related genes, NRT2 and NAR2, and nitrate uptake during the nitrate induction. Four rice NRT2S (OSNRT2.1 2.4) and two NAR2s (OsNAR2.1 2.2) were identified in the rice genome database. We analyzed the expression of the genes in the roots and shoots after supply of nitrate with or without ammonium pretreatment by the RT-PCR method. The apparent nitrate uptake was also measured. Differential expression of OsNRT2.1 and OsNRT2.2, which have the same ORF with different $5^{\prime}$ - and $3^{\prime}$-UTR, was observed in the seedlings pretreated with ammonium. The apparent nitrate uptake synchronously increased after supply of nitrate with the expression pattern of OSNRT2.1 in roots either with or without ammonium pretreatment. The expression of OSNAR2.1, which is thought to be an activator gene for the function of NRT2, was also coordinated with that of OsNRT2.1. On the other hand, OsNRT2.2 was already expressed after ammonium pretreatment, suggesting that OsNRT2.2 would be transcribed to take up nitrate in flooded soils. The present results indicate that OsNRT2.1, OSNRT2.2 and OSNAR2.1 are candidate genes when breeding for efficient use of nitrate in rice.
\end{abstract}

Key Words: High-affinity nitrate transporter, NRT2, NAR2, Oryza sativa, nitrate induction, nitrate uptake.

\section{Introduction}

Breeding of crops that utilize nitrogen efficiently is one of the important goals of crop breeding, because nitrogen is the most important factor limiting growth or yield in crops. For most cultivated crops, nitrate is the major nitrogen source. In rice (Oryza sativa L.), the nitrate produced by nitrification has been shown to be taken up with the diffusion of oxygen through the roots in flooded soils (Kirk and Kronzucker 2005). In addition, rice grows better and utilizes nitrogen efficiently when nitrate and ammonium are supplied together (Ta and Ohira 1981, Kronzucker et al. 1999b). It is more important to study nitrate uptake than generally thought. On the other hand, the progress in the analysis of the rice genome (Sasaki et al. 2002, Sasaki et al. 2005, Yuan et al. 2005) has revealed that there is extensive synteny and gene homology between rice and cereal crops (Goff et al. 2002), indicating that rice can be a model plant for studying nitrate uptake mechanisms at the molecular level. Therefore, it is useful to reveal the regulatory mechanism of nitrate uptake in rice.

Nitrate uptake is mediated by the low affinity transport system (LATS) and high-affinity transport system (HATS) (Crawford and Glass 1998, Forde 2000), which are encoded

Communicated by Andris Kleinhofs

Received December 9, 2005. Accepted April 11, 2006.

*Corresponding author (e-mail: hasegawa@ses.usp.ac.jp) by NRT1 and NRT2, respectively (Tsay et al. 1993, Krapp et al. 1998, Vidmar et al. 2000a, Cerezo et al. 2001, Filleur et al. 2001). HATS consists of two systems, the nitrateinducible HATS (iHATS) and the constitutive HATS (cHATS) (Wang and Crawford 1996, Crawford and Glass 1998, Forde 2000). In Arabidopsis thaliana, seven genes were isolated as NRT2 family gene members (Orsel et al. $2002 \mathrm{~b}$ ), suggesting that NRT2 in higher plants is composed of several genes. The mechanism of iHATS for nitrate at the molecular level has been revealed by functional analysis using $A$. thaliana mutant, atnrt2, which is disrupted in A. thaliana NRT2.1 (AtNRT2.1) and in a part of AtNRT2.2 and showed a decrease of nitrate uptake ability only in iHATS (Cerezo et al. 2001, Filleur et al. 2001). However, the functional roles for the other members of NRT2 remain uncertain. In rice, although the function of NRT1 has been revealed (Tsay et al. 1993, Lin et al. 2000), investigations of NRT2 have not been carried out. To develop efficient use of nitrogen in rice, it is important to reveal the roles of NRT2 family genes.

To understand the role of the NRT2 family we need to investigate the relationship between the expression of NRT2 and nitrate uptake during the period of nitrate induction (Amarasinghe et al. 1998, Krapp et al. 1998, Zhuo et al. 1999, Vidmar et al. 2000a). By the relative quantitative RTPCR method, AtNRT2 family genes have been grouped into three categories, nitrate-inducible, nitrate-repressible and nitrate-constitutive (Okamoto et al. 2003). Moreover, there 
is a clear positive correlation between nitrate uptake and the amounts of AtNRT2.1 transcripts in nitrate induction (Zhuo et al. 1999, Okamoto et al. 2003).

The differential repression of transcripts of NRT2 family genes except for nitrate constitutive genes, 3 to $12 \mathrm{~h}$ after supply of nitrate has been reported in barley (Hordeum vulgare) and A. thaliana (Vidmar et al. 2000a, Okamoto et al. 2003). However, most NRT2 family genes are induced coordinately by the nitrate treatment (Vidmar et al. 2000a, Okamoto et al. 2003). The negative feedback regulation for the expression of NRT2 and nitrate uptake has been reported to be caused by the metabolites downstream, ammonium or glutamine (Youngdahl et al. 1982, Henriksen and Spanswick 1993, King et al. 1993, Quesada et al. 1997, Kronzucker et al. 1999a, Lejay et al. 1999, Zhuo et al. 1999, Vidmar et al. 2000b, Glass et al. 2002, Orsel et al. 2002a). Therefore, the differential expression patterns of NRT2s may be attributed to a difference in the response to nitrogen status among the NRT2 family members.

Furthermore, it is necessary to consider the activation and/or regulation factors for NRT2 function at the molecular level. In Chlamydomonas reinhardtii, NAR2 gives NRT2 the function of transporting nitrate through the membrane (Quesada et al. 1994, Zhou et al. 2000). Recently, Tong et al. (2005) found NAR2-like genes of $C$. reinhardtii in barley and reported that one of the barley NAR2s (HvNAR2.1 2.3), HvNAR2.3, could give functional nitrate transport activity to HvNRT2.1 in the Xenopus laevis oocyte. This result indicated that $N A R 2 s$ are also important for the function of NRT2 with nitrate uptake in higher plants.

In this study, to reveal the regulation of HATS for nitrate at the molecular level in rice, we focused on the induction of nitrate uptake in relation to the time-course induction patterns of OsNRT2 family genes and OsNAR2 genes with or without ammonium pretreatment, based on the hypothesis that there is a difference in the response to nitrogen status among NRT2 family genes.

\section{Materials and Methods}

\section{Plant materials and cultivation}

Rice (Oryza sativa L. cv. Nipponbare) seeds were surface-sterilized for 30 min with $10 \% \mathrm{H}_{2} \mathrm{O}_{2}$, and rinsed with de-ionized water twice. The seeds were germinated and cultured with de-ionized water in a biotron (LH-5-1205, Nippon Medical and Chemical Instruments, Osaka, Japan) controlled at $25 \pm 1^{\circ} \mathrm{C}$ under continuous lighting $(70.9 \mu \mathrm{mol}$ $\mathrm{m}^{-2} \mathrm{~s}^{-1}$ ) with fluorescent lamps (FL20SS W/18, Toshiba, Tokyo, Japan) for 3 weeks.

Isolation and analysis of OsNRT2s and OsNAR2s from rice genome database

Four rice NRT2 family genes (OsNRT2.1 2.4) were extracted from the rice genome database (Sasaki et al. 2002, Sasaki et al. 2005) using DDBJ BLAST search (Altschul et al. 1997, Tateno et al. 2005) and BLAST search system in the TIGR rice genome annotation (Yuan et al. 2005: http:// rice.tigr.org/) with registered rice high-affinity nitrate transporter gene sequence (OsNRT2: GenBank accession No. AB008519). Extracted DNA sequences were analyzed with the TIGR rice genome annotation and Gramene (Jaiswal et al. 2002, Ware et al. 2002: http://www.gramene.org/) to determine the location of each OSNRT2 on rice chromosomes. Two NAR2s in rice were also extracted from the TIGR rice genome annotation using the amino acid sequence of HvNAR2.3 (GenBank accession No. AAP31852). Plant cisacting regulatory DNA elements in $O_{s N R T 2 s}$ and $O_{s N A R} s$ were searched with PLACE (Higo et al. 1999). Transmembrane spanning regions of each OsNRT2 and OsNAR2 were determined using TMpred (Hofmann and Stoffel 1993), TopPred (von Heijne 1992, Claros and von Heijne 1994), MEMSAT (Jones et al. 1994, McGuffin et al. 2000) and HMMTOP (Tusnády and Simon 1998, Tusnády and Simon 2001). ClustalW was used for the phylogenic analysis of NRT2s (Thompson et al. 1994) in DDBJ and Tree View (Page 1996).

\section{Analysis of expression of OsNRT2s and OsNAR2s}

For the molecular analysis, 3-week-old seedlings were treated with $200 \mu \mathrm{M} \mathrm{KNO}_{3}$ containing $400 \mu \mathrm{M} \mathrm{CaSO}_{4}$ solution after pretreatment with $400 \mu \mathrm{M} \mathrm{CaSO}_{4}$ or $500 \mu \mathrm{M}$ $\left(\mathrm{NH}_{4}\right)_{2} \mathrm{SO}_{4}$ containing $400 \mu \mathrm{M} \mathrm{CaSO}$ for $20 \mathrm{~h}$. Total RNA was extracted with TRI reagent ${ }^{\mathbb{R}}$ (Molecular Research Center, INC, Cincinnati, OH, USA) from the roots and shoots of 10 seedlings. Extracted total RNAs were treated with DNase (TaKaRa, Otsu, Japan) according to the manufacturer's manual. Total RNA concentrations were determined by UV spectrophotometry (FAS-III full system, TOYOBO, Osaka, Japan). First strand cDNA synthesis using RevaTra Ace ${ }^{\circledR}$ (TOYOBO, Osaka, Japan) was performed with $1 \mu \mathrm{g}$ aliquots of total RNA and oligo dT(20) primer according to the manual.

Specific primers for each OsNRT2 were as follows: 5'GCTAGTAGTTGCCATGGACTCG-3' and 5'-ATCAGGA TGTTCCCCGTTTG-3' for OsNRT2.1; 5'-TGGAGCGAAC AGGAGAAGAG-3' and 5'-GCCTCTTAATTAGGCGTGC TC-3' for OsNRT2.2; 5'-GCCATCCACAAGATCGGTAG$3^{\prime}$ and TGTGGAGCTTCCCGTAGTTG-3' for OsNRT2.3; 5'-ATCGTTCCCTTCGTCTGCAA-3' and 5'-TACCTGGA CCCGCTGAAGAA-3' for OsNRT2.4. OsNAR2.1 and OsNAR2.2 specific primers were as follows: 5'-AGGTGTT CCTCTCCAAGCCC-3' and 5'-CTTCACGCTCTTGAAC GCCG-3' for OsNAR2.1; 5'-CCTCGAGAACAAGAAGAA GAACAAGTGA-3' and 5'-TGCTGGGCGCTGTAGACAC$3^{\prime}$ for OsNAR2.2. Specific primers for Actin, 5'-CTTCATA GGAATGGAAGCTGCGGGTA-3' and 5'-CGACCACCTT GATCTTCATGCTGCTA-3' (McElroy et al. 1990), were used for the internal control as demonstrated by Sonoda et al. (2003).

The program of PCR was $95^{\circ} \mathrm{C}$ for 1 min for 1 cycle followed by $95^{\circ} \mathrm{C}$ for $5 \mathrm{sec}$ and $60^{\circ} \mathrm{C}$ for $20 \mathrm{sec}$ for $26 \sim 32$ cycles as an amplification step. In addition, OsNRT1.1 
(GenBank accession No. AF140606) were amplified using specific primers, 5'-ATCAGGATGTTCCCCGTTTG-3' and 5'-GCCGATGTGTTTCTCCATCA-3', for comparison with the results of OsNRT2s expression patterns. The amplified DNA fragments were checked by electrophoresis on $3 \%$ (w/v) Certified ${ }^{\mathrm{TM}}$ Low Range Ultra Agarose (Bio-Rad, Hercules, CA, USA) and sequenced with an ABI Prism 310 Genetic Analyzer (Applied Biosystems, Foster City, CA, USA) to confirm their identity. The gene expression in root and shoot was analyzed periodically 3 times.

\section{Apparent nitrate uptake measurement}

Seedlings cultivated for 3 weeks in de-ionized water were treated with $400 \mu \mathrm{M} \mathrm{CaSO}_{4}$ solution with or without $500 \mu \mathrm{M}\left(\mathrm{NH}_{4}\right)_{2} \mathrm{SO}_{4}$ for $20 \mathrm{~h}$ (pretreatment). Then 10 seedlings were transferred into the $100 \mathrm{ml}$ solution containing $200 \mu \mathrm{M}$ $\mathrm{KNO}_{3}$ and $400 \mu \mathrm{M} \mathrm{CaSO}_{4}$ (experimental solution). The solution of $1.8 \mathrm{ml}$ was taken up at the given times for $24 \mathrm{~h}$ after transferring to the experimental solution. Nitrate concentration was measured with an ion-chromatograph (DX-3000, Dionex, Sunnyvale, CA, USA) equipped with an anionspecific column (IonPacR AS12A, Dionex, Sunnyvale, CA, USA). Amounts of apparent nitrate taken up from the experimental solution were evaluated based on the nitrate uptake index as nitrate uptake taken up from the solution per fresh weight of 10 whole seedlings. Nitrate uptake was calculated from the depletion of experimental solution (Hasegawa and Ichii 1994, Araki et al. 2005). The experiment was repeated four to six times and five replicates were used in each treatment.

\section{Results}

\section{Information of OsNRT2s}

Four OsNRT2 (OsNRT2.1 OsNRT2.4) sequences were extracted from the rice genome database using the BLAST search on DDBJ and TIGR rice genome annotation and Gramene (Table 1). The sequences of OsNRT2.1, OsNRT2.2 and OsNRT2.3 have been registered as putative NRT2 cDNAs (GenBank accession numbers: AB008519, AK109733 and AK109776, respectively). Interestingly, OsNRT2.1 and OsNRT2.2 had the same ORF but with different sequences of 5'- and 3'-UTR. Neither OsNRT2.1 nor OsNRT2.2 had an intron. In addition, OsNRT2.1 and OsNRT2.2 were found on chromosome 2 in the region of $655,324 \sim 668,962 \mathrm{bp}$, holding only a gene encoding hypothetical protein between OsNRT2.1 and OsNRT2.2, being in the forward and the inverse direction, respectively. OSNRT2.3 and OsNRT2.4 were found on chromosome 1 (the distance between two genes is about 9,000,000 bp) (Table 1). Several putative TATA boxes were found in OsNRT2.1, OsNRT2.2 and OsNRT2.4. TATABOX2 (Grace et al. 2004) was found at -152 and at -124 in $O s N R T 2.1$ and $O s N R T 2.2$, respectively. TATABOXOSPAL (Zhu et al. 2002) was found at -156 in OsNRT2.4. In OsNRT2.3, a putative functional TATA box motif was not found.

The four OsNRT2s had the conserved amino acid motifs of [AG]-G-[WL]-G-N-M-G (Trueman et al. 1996), R-[PA]-X-G-G-X-X-[SA]-D and F-G-M-R-G-R-L-W (Okamoto et al. 2003) (Fig. 1). Membrane spanning regions of each OsNRT2 were predicted 11 to 12 times using TopPred, MEMSAT and TMpred. MEMSTAT revealed 12 trans-membrane regions in all OsNRT2s (Fig. 1). These data indicated that the extracted sequences are genes encoding a high-affinity rice nitrate transporter.

The phylogenic analysis for NRT2s based on the amino acid sequence demonstrated that OsNRT2.1 and OsNRT2.2 belonged to a monocotyledonous NRT2 cluster, while OsNRT2.3 and OsNRT2.4 belonged to another NRT2 cluster including a lower organism NRT2 group (Fig. 2) as AtNRT2.5 and AtNRT2.7. OsNRT2.3 and OsNRT2.4 have $58.3 \%$ and $43.3 \%$ similarity to OsNRT2.1, respectively. OsNRT2.1 and OsNRT2.2 showed higher similarity to the other higher plants NRT2.1 than OsNRT2.3 or OsNRT2.4 (data not shown, refer to Fig. 2).

\section{OsNAR2.1 and OsNAR2.2}

Two OsNAR2 genes were also extracted from the rice genome database (Table 1). OsNAR2.1 and OsNAR2.2 were located in chromosomes 2 and 4, respectively. A putative TATA box was found at -84 as TATABOX2 in OsNAR2.1 and that at -33 as TATABOX4 (Grace et al. 2004) in OsNAR2.2. The deduced amino acid sequence of OsNAR2.1 had $62.0 \%$ similarity to OsNAR2.2. OsNAR2.1 and OsNAR2.2 had $59.2 \%$ and $63.2 \%$ similarity to HvNAR2.3, respectively. The similarities of two OsNAR2s to several NAR2s of other higher plants were from $42.6 \%$ to $65.5 \%$. OsNAR2.1 and OsNAR2.2 had 1 to 3 time(s) transmembrane region(s) predicted by TMpred, TopPred and HMMTOP. MEMSAT predicted a 1 time trans-membrane

Table 1. Features of $N R T 2 s$ and $N A R 2 s$ in rice

\begin{tabular}{lcccc}
\hline \hline Gene & Chromosome & Strand (+/-) & Accession No. & Gene ID \\
\hline OsNRT2.1 & 2 & + & AB008519 & LOC_Os02g02170.1 \\
OsNRT2.2 & 2 & - & AK109733 & LOC_Os02g02190.1 \\
OsNRT2.3 & 1 & + & AK109776 & LOC_Os01g50820 \\
OsNRT2.4 & 1 & - & - & LOC_Os01g36720 \\
\hline OsNAR2.1 & 2 & - & - & LOC_Os02g38230 \\
OsNAR2.2 & 4 & - & - & LOC_Os04g40410 \\
\hline
\end{tabular}




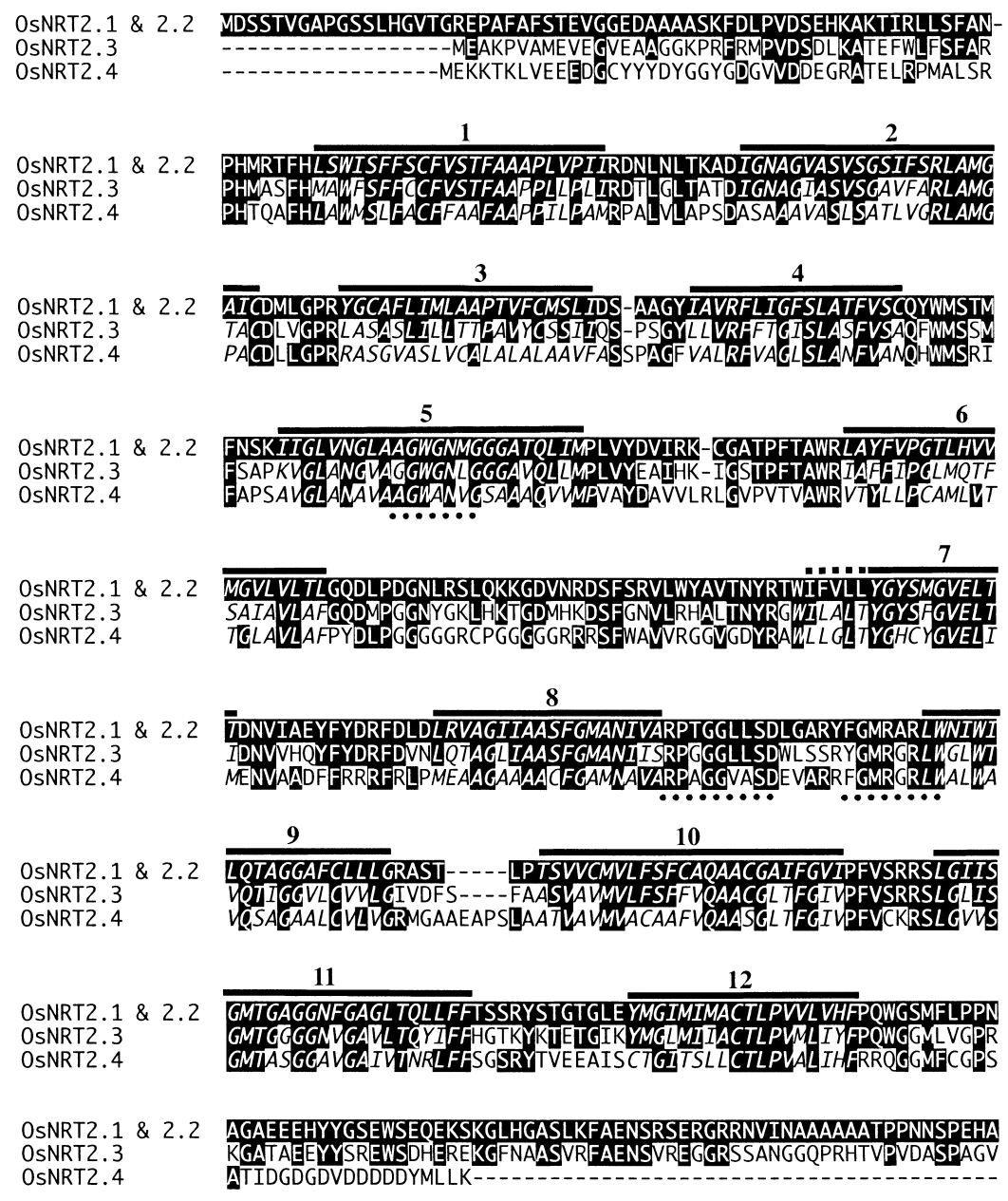

Fig. 1. Deduced amino acid sequences of OsNRT2 family (OsNRT2.1 2.4). Sequences were aligned with ClustalW. Shaded region shows the amino acid sequence homology to OsNRT2.1. Numbered lines above the sequences indicate predicted trans-membrane spanning regions, determined using MEMSTAT. Each OsNRT2 trans-membrane spanning region is indicated by italics. Conserved sequence regions (as described in results) are indicated by dotted lines under the sequences. Gaps are indicated by hyphens.

region for each OsNAR2.1 and OsNAR2.2.

\section{OsNRT2s and OsNAR2s expression in roots}

Pretreatment with $1 \mathrm{mM}$ ammonium affected the expression of OsNRT2s. In the seedlings without ammonium pretreatment, transcripts of OsNRT2.1, OsNRT2.2 and OSNRT2.4 were detected from $0.5 \mathrm{~h}$ after the onset of 200 $\mu \mathrm{M}$ nitrate treatment, while OsNRT2.3 was already expressed before the nitrate induction (Fig. 3). When the seedlings were pretreated with ammonium, the induction of OsNRT2.1 transcription was impaired from 0 to $2 \mathrm{~h}$ after the beginning of nitrate treatment (Fig. 3). The transcription of OsNRT2.1 was increased from $2 \mathrm{~h}$ after the beginning of nitrate treatment but the transcript abundance of OsNRT2.1 declined after $24 \mathrm{~h}$. On the other hand, OsNRT2.2 was expressed at the beginning of nitrate treatment following pretreatment with ammonium, and remained at elevated levels for the duration of nitrate treatment. OsNRT2.3 transcripts were already detected from beginning of nitrate induction. The expression pattern of OsNRT2.4 was similar to that of OsNRT2.1 (Fig. 3).

Nitrate induced the expression of OSNAR2.1 within 0.5 $\mathrm{h}$ after transferring the seedlings to nitrate from an $\mathrm{N}$-free solution, as it did OsNRT2.1 and OsNRT2.4 (Fig. 3). However, the expression level of $O s N A R 2.1$ at $0.5 \mathrm{~h}$ was lower than that of OSNAR2.1 treated with nitrate for $1 \mathrm{~h}$ or more (Fig. 3). On the other hand, OsNAR2.2 was expressed at the beginning of the treatment with nitrate from $\mathrm{N}$-starvation. In the seedlings pretreated with ammonium, the expression of OsNAR2.1 and OsNAR2.2 was not clearly detected for $0.5 \mathrm{~h}$ after transfer to nitrate.

OsNRT1.1, which is involved only in LATS for nitrate (Lin et al. 2000), was constitutively expressed in roots under nitrate concentrations below $200 \mu \mathrm{M}$. 


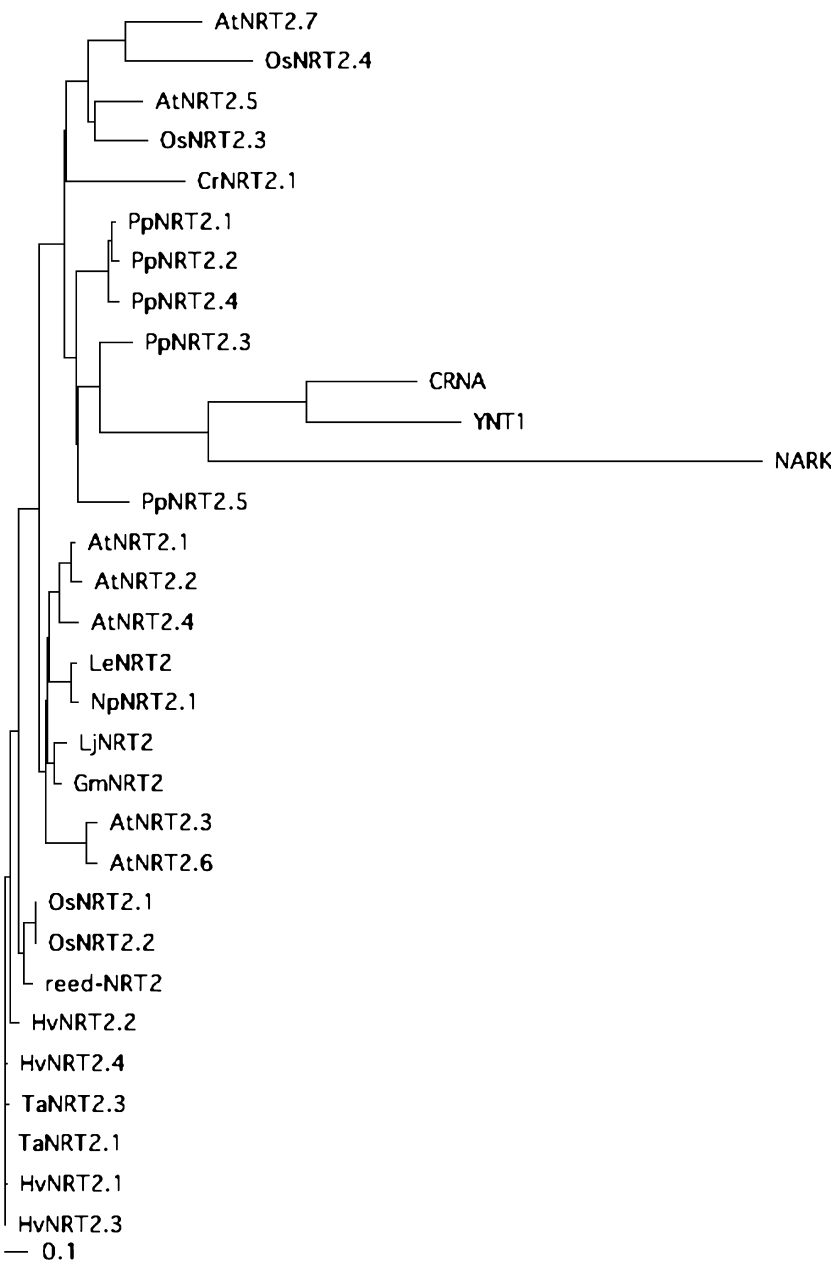

Fig. 2. Phylogenic analysis of NRT2 proteins. Deduced amino acid sequences of NRT2s were aligned by ClustalW, and Tree View was used for establishing the phylogenic tree. Protein ID numbers are Oryza sativa OsNRT2.1, O82138; OsNRT2.2, Q6ZH34; OsNRT2.3, Q94JG1; OsNRT2.4, BAB90798; Arabidopsis thaliana AtNRT2.1, O82811; AtNRT2.2, O82412; AtNRT2.3, Q9FJH7; AtNRT2.4, Q9FJH8; AtNRT2.5, Q9LPV5; AtNRT2.6, Q9LXH0; AtNRT2.7, Q9LYK2; Hordeum vulgare HvNRT2.1, P93182; HvNRT2.2, P93183; HvNRT2.3, Q9XEG4; HvNRT2.4, Q9XEG5; Triticum aestivum TaNRT2.1, Q9ATG9; TaNRT2.3, Q940R8; Phragmites australis reed NRT2, Q84LK4; Glycine max GmNRT2, O64999; Lotus japonicus LjNRT2, Q9ARC5; Nicotiana plumbaginifolia NpNRT2.1, O04431; NtNRT2.2, CAD89799; Lycopersicon esculentum LeNRT2.1, Q9SQG6; Physcomitrella patens PpNRT2.1, Q76C06; PpNRT2.2, Q76C05; PpNRT2.3, Q76C04; PpNRT2.4, Q76C03; PpNRT2.5, Q76C02; Chlamydomonas reinhardtii CrNRT2.1, Q39608; Hansenula polymorpha YNT1, Q92240; Aspergillus nidulans CRNA, Q5BEM2; Escherichia coli NARK, P10903.

OsNRT2s and OsNAR2s expression in shoots

$O s N R T 2 s$ and $O s N A R 2 s$ were also expressed in shoots (Fig. 4), but not as much as in roots. In particular, the expression level of OSNRT2.1 in shoots was very low either with or without ammonium pretreatment (Fig. 4). OsNRT2.2 and $O S N R T 2.4$ were expressed after the nitrate treatment

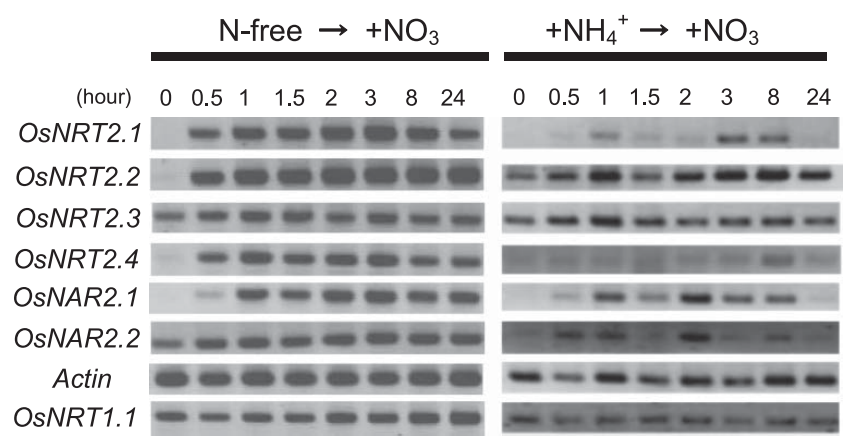

Fig. 3. Expression patterns of $O s N R T 2 s$ and $O s N A R 2 s$ in roots from the beginning of nitrate treatment. Seedlings that had been pretreated with ammonium $\left(+\mathrm{NH}_{4}{ }^{+}\right)$or without ammonium $(\mathrm{N}-$ free) were treated with nitrate. The total RNAs were extracted from the seedlings sampled at the given times (hour), indicated by the numbers in the upper row, after nitrate treatment. Actin was the internal control gene. OsNRT1.1 was used for comparison with the results of $O S N R T 2 s$ expression patterns. This amplification step was repeated until signals were clear: 32 cycles for OsNRT2s, 30 cycles for OsNAR2s and 26 cycles for Actin.

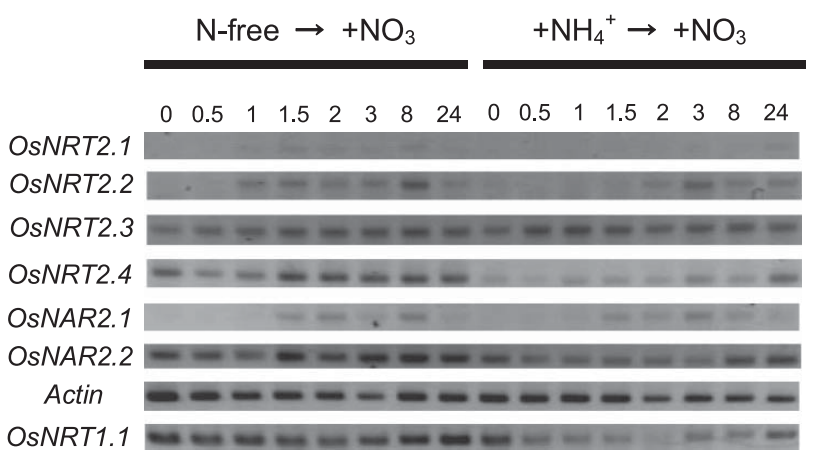

Fig. 4. Expression patterns of $O S N R T 2 s$ and $O S N A R 2 s$ in shoots from the beginning of nitrate treatment. The procedures were the same as those in Fig. 3 except that the amplification step was repeated 30 cycles for Actin.

without ammonium pretreatment. OsNRT2.2 was expressed from $1 \mathrm{~h}$ after the beginning of nitrate treatment, and the transcript abundance of OSNRT2.2 peaked at $8 \mathrm{~h}$ then declined to a barely detectable level at $24 \mathrm{~h}$ (Fig. 4). However, in the seedlings pretreated with ammonium, no transcripts of OsNRT2.2 were detected at 0 to $1.5 \mathrm{~h}$. OSNRT2.3 was expressed in shoots, as in roots (Fig. 3 and Fig. 4). The expression of OSNRT2.4 in shoots was similar to that of OSNRT2.1 as in roots.

In shoots, OSNAR2.1 was expressed slightly, while OsNAR2.2 was constitutively expressed (Fig. 4). OsNRT1.1 was also constitutively expressed in shoots (Fig. 4).

\section{Apparent nitrate uptake}

A difference in the apparent nitrate uptake index was observed between the seedlings pretreated with ammonium and those without pretreatment. When the seedlings were transferred to a nitrate solution from an $\mathrm{N}$-free solution, the nitrate uptake index increased linearly following a $0.5 \mathrm{~h}$ 


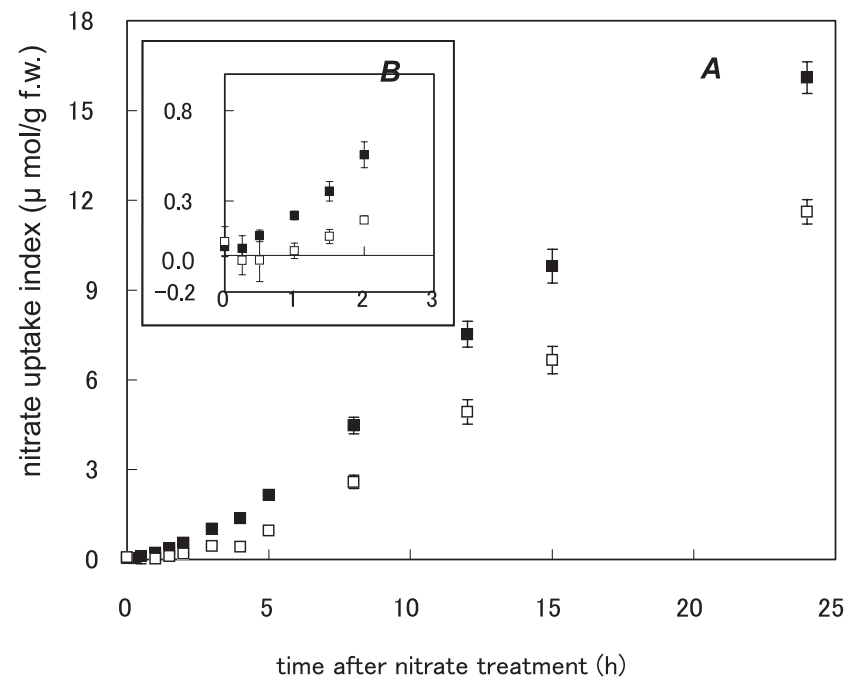

Fig. 5. Increase in apparent nitrate uptake index from $200 \mu \mathrm{M} \mathrm{KNO}_{3}$ solution with time (A and $\mathrm{B}$ ). The seedlings were immersed in the nitrate solution after pretreatment for $20 \mathrm{~h}$ with or without $500 \mu \mathrm{M}\left(\mathrm{NH}_{4}\right)_{2} \mathrm{SO}_{4}$. Open and closed squares indicate the amounts of nitrate uptake after pretreatment with $\mathrm{NH}_{4}{ }^{+}$and that without $\mathrm{NH}_{4}{ }^{+}$, respectively. A shows the data during the nitrate treatment for $24 \mathrm{~h}$ after the beginning of nitrate treatment. B shows the data from $0 \mathrm{~h}$ to $2 \mathrm{~h}$. Points and bars are means and SE of four to six replications.

induction period (Fig. 5B) with increase in treatment duration. Apparent nitrate uptake was not clearly detected until $1.5 \mathrm{~h}$ after transfer to nitrate from an ammonium solution (Fig. 5B), suggesting an induction period of about $1 \mathrm{~h}$ for the uptake. After $1.5 \mathrm{~h}$ from the beginning of nitrate treatment, nitrate uptake index increased linearly with the prolongation of nitrate treatment (Fig. 5A).

\section{Discussion}

Of the four NRT2 family genes extracted from the rice genome database, three genes were nitrate inducible and one gene was expressed before the nitrate induction. In roots, nitrate induced the expression of both OsNRT2.1 and OsNRT2.2 and the apparent nitrate uptake within $0.5 \mathrm{~h}$ after $\mathrm{N}$-starvation (Fig. 3). The amounts of nitrate uptake increased with prolongation of nitrate treatment (Fig. 5). This short induction period of nitrate uptake in rice is coincident with previous reports (Sasakawa and Yamamoto 1978, Hasegawa et al. 1989, Kronzucker et al. 2000). Nitrate induction occurred more rapidly in rice than in barley, which is known as a highly efficient nitrate user (reviewed in Kirk and Kronzucker 2005). On the other hand, the time-lag of apparent nitrate uptake induction in the seedlings pretreated with ammonium (Fig. 5) could be caused by transient negative feedback regulation.

An interesting and important feature found is that OsNRT2.1 and OsNRT2.2 have the same ORF with different sequences of 5'-UTR and 3'-UTR and their expression in response to nitrogen status are different in planta. The expres- sion patterns of OsNRT2.1 and OsNRT2.2 after transfer of the seedlings to nitrate solution from $\mathrm{N}$-free solution were similar. Therefore, OsNRT2.1 and OsNRT2.2 are both nitrate-inducible genes as mentioned by Okamoto et al. (2003). However, the expression patterns in the seedlings pretreated with ammonium were different, suggesting a difference in the response to the negative feedback regulation by ammonium pretreatment between OsNRT2.1 and OsNRT2.2. As OsNRT2.1 and OsNRT2.2 encode the same amino acid sequence, it is difficult to assess the contribution of their gene products for nitrate uptake. In several higher plants, nitrate uptake has been shown to be positively correlated with the amounts of NRT2 transcripts in nitrate induction (Krapp et al. 1998, Amarasinghe et al. 1998, Zhuo et al. 1999, Vidmar et al. 2000a, Okamoto et al. 2003). As the OsNRT2.1 was expressed in parallel with the increase of apparent nitrate uptake index, it is concluded that OsNRT2.1 is a key gene for the induction of nitrate uptake in rice. However, the result of the gene expression analysis implied that the products of OsNRT2.2 also play an important role in nitrate uptake in the presence of nitrate and ammonium.

Nitrate has been proposed to be a signal molecule for transcription of genes related to nitrate assimilation (Crawford 1995, Wang et al. 2004). The difference in the expression of OsNRT2.1 and OsNRT2.2 might be attributed to the 5'-UTR up-stream region. Although, at present, the nitrate signal transduction pathway and its transcription factor in higher plants are still unclear, the high nitrogen status produced by ammonium pretreatment probably influences the transcription pathway of OSNRT2.1 even where nitrate could be perceived as a signal by the seedlings.

The functional nitrate transport mediated by NRT2 has been shown to require the product of NAR2 in C. reinhardtii and barley using the Xenopus oocyte expression system (Quesada et al. 1994, Zhou et al. 2000, Tong et al. 2005). The expression of OSNAR2.1 was induced by nitrate treatment and repressed by ammonium pretreatment as demonstrated in OsNRT2.1. In addition, the expression of OsNAR2.1 was clearly associated with an increase in the apparent nitrate uptake following pretreatment with or without ammonium (Fig. 5). However, the expression of OSNAR2.1 and $O s N R T 2.2$ did not show a synchronous pattern when the seedlings were pretreated with ammonium (Fig. 3). These results suggest that $O S N A R 2.1$ is also one of the key genes in nitrate uptake in rice.

The difference in gene expression between OsNRT2.1 and OsNRT2.2 was also found in shoots. The transcripts of OsNRT2.1 were barely detectable (Fig. 4), suggesting that OsNRT2.1 is a root-specific gene. On the other hand, the expression of OSNRT2.2 was induced by nitrate treatment, indicating that nitrate induction occurred in shoots (Fig. 4). Unlike the expression in roots, the repression of the OsNRT2.2 expression by ammonium pretreatment was found in shoots. OsNAR2.1 was barely detectable and expressed synchronously with OsNRT2.1 in shoots, suggesting that $O S N A R 2.1$ is also a root-specific gene. 
Differentiation of the NRT2 family was demonstrated in rice as shown in the AtNRT2 family (Fig. 2) (Orsel et al. 2002b). Phylogenic analysis demonstrated that OsNRT.2.1 and OsNRT2.2 were categorized in a monocotyledonous group. On the other hand, OsNRT2.3 and OsNRT2.4 were categorized in other NRT2 clusters including AtNRT2.5 and AtNRT2.7 (Orsel et al. 2002b), which are nitrate-repressible and constitutively expressed genes, respectively (Okamoto et al. 2003). OsNRT2.3, expressed constitutively, affected neither the nitrate uptake nor expression of OSNRT2.4 in roots and shoots. Therefore, OsNRT2.3 and OsNRT2.4 are considered to be minor high-affinity nitrate transporters in rice.

In conclusion, it is important to consider OSNAR2.1, OsNRT2.1 and OsNRT2.2 when breeding for efficient use of nitrogen in rice. Further investigations on OsNRT2.2 are necessary to understand the regulation of nitrate uptake mechanisms in rice.

\section{Acknowledgments}

We are grateful to Dr. K. Sueyoshi (Faculty of Agriculture, Niigata University, Japan) for supplying valuable information for this study. We thank Dr. T. Irie (School of Environmental Science, The University of Shiga Prefecture, Japan) for critical reading of the manuscript.

\section{Literature Cited}

Altschul, S.F., T.L. Madden, A.A. Schaffer, J. Zhang, Z. Zhang, W.Miller and D.J.Lipman (1997) Gapped BLAST and PSIBLAST: a new generation of protein database search programs. Nucleic Acids Res. 25: 3389-3402.

Amarasinghe, B.H.R.R., G.L.deBruxelles, M.Braddon, I.Onyeocha, B.G.Forde and M.K.Udvardi (1998) Regulation of GmNRT2 expression and nitrate transport activity in roots of soybean (Glycine max). Planta 206: 44-52.

Araki, R., M.Mori, M.Mori and H.Hasegawa (2005) Genetic differences in nitrate uptake in two clones of the common reed, Phragmites australis. Breed. Sci. 55: 297-302.

Cerezo, M., P.Tillard, S.Filleur, S. Muños, F. Daniel-Vedele and A.Gojon (2001) Major alterations of the regulation of root $\mathrm{NO}_{3}{ }^{-}$uptake are associated with the mutation of $\mathrm{Nrt2.1}$ and Nrt2.2 genes in Arabidopsis. Plant Physiol. 127: 262-271.

Claros, M.G. and G.vonHeijne (1994) TopPred II: an improved software for membrane protein structure predictions. CABIOS 10: 685-686.

Crawford,N.M. (1995) Nitrate: nutrient and signal for plant growth. Plant Cell 7: 859-868.

Crawford,N.M. and A.D.M.Glass (1998) Molecular and physiological aspects of nitrate uptake in plants. Trends Plant Sci. 3: 389395.

Filleur,S., M.-F.Dorbe, M.Cerezo, M.Orsel, F.Granier, A.Gojon and F.Daniel-Vedele (2001) An Arabidopsis T-DNA mutant affected in Nrt2 genes is impaired in nitrate uptake. FEBS Lett. 489: 220-224.

Forde,B.G. (2000) Nitrate transporters in plants: structure, function and regulation. Biochim. Biophys. Acta 1465: 219-235.
Goff,S.A., D.Ricke, T.-H.Lan, G.Presting, R.Wang et al. (2002) A draft sequence of the rice genome (Oryza sativa L. spp. japonica). Science 296: 92-100.

Glass,A.D.M., D.T.Britto, B.N.Kaiser, J.R.Kinghorn, H.J.Kronzucker, A.Kumar, M.Okamoto, S.Rawat, M.Y.Siddiqi, S.E.Unkles and J.J.Vidmar (2002) The regulation of nitrate and ammonium transport systems in plants. J. Exp. Bot. 53: 855-864.

Grace,M.L., M.B.Chandrasekharan, T.C.Hall and A.J.Crowe (2004) Sequence and spacing of TATA box elements are critical for accurate initiation from the $\beta$-phaseolin promoter. J. Biol. Chem. 279: 8102-8110.

Hasegawa,H., M.Kashiyama, T.Korekata and H.Watanabe (1989) Measurement of nitrate uptake by rice seedlings using nitratespecific electrode. Rep. Soc. Crop Sci. Breed. Kinki, Japan 34: 39-42 (in Japanese).

Hasegawa,H. and M.Ichii (1994) Variation in Michaelis-Menten kinetic parameters for nitrate uptake by the young seedlings in rice (Oryza sativa L.). Breed. Sci. 44: 383-386.

von Heijne, G. (1992) Membrane protein structure prediction: Hydrophobicity analysis and the 'Positive Inside' rule. J. Mol. Biol. 225: 487-494.

Henriksen, G.H. and R.M. Spanswick (1993) Investigation of the apparent induction of nitrate uptake in barley (Hordeum vulgare L.) using $\mathrm{NO}_{3}{ }^{-}$-selective microelectrodes. Plant Physiol. 103: 885-892.

Higo, K., Y.Ugawa, M.Iwamoto and T.Korenaga (1999) Plant cisacting regulatory DNA elements (PLACE) database: 1999. Nucleic Acids Res. 27: 297-300.

Hofmann, K. and W.Stoffel (1993) TMbase - A database of membrane spanning proteins segments. Biol. Chem. Hoppe-Seyler 374: 166.

Jaiswal, P., D. Ware, J.Ni, K.Kuan, W.Zhao, S.Schmidt, X.Pan, K.Clark, L.Teytelman, S.Cartinhour, L.Stein and S.McCouch (2002) Gramene: development and integration of trait and gene ontologies for rice. Comp. Funct. Genom. 3: 132-136.

Jones,D.T., W.R.Taylor and J.M.Thornton (1994) A model recognition approach to the prediction of all-helical membrane protein structure and topology. Biochemistry 33: 3038-3049.

King, B.J., M.Y.Siddiqi, T.J.Ruth, R.L.Warner and A.D.M.Glass (1993) Feedback regulation of nitrate influx in barley roots by nitrate, nitrite, and ammonium. Plant Physiol. 102: 1279-1286.

Kirk, G.J.D. and H.J.Kronzucker (2005) The potential for nitrification and nitrate uptake in the rhizosphere of wetland plants: Modeling study. Ann. Bot. 96: 639-646.

Krapp,A., V.Fraisier, W.-R.Scheible, A.Quesada, A.Gojon, M.Stitt, M.Caboche and F.Daniel-Vedele (1998) Expression studies of $N r t 2: 1 N p$, a putative high-affinity nitrate transporter: evidence for its role in nitrate uptake. Plant J. 14: 723-731.

Kronzucker,H.J., A.D.M.Glass and M.Y.Siddiqi (1999a) Inhibition of nitrate uptake by ammonium in barley. Analysis of component fluxes. Plant Physiol. 120: 283-291.

Kronzucker,H.J., M.Y.Siddiqi, A.D.M.Glass and G.J.D.Kirk (1999b) Nitrate-ammonium synergism in rice. A subcellular flux analysis. Plant Physiol. 119: 1041-1045.

Kronzucker,H.J., A.D.M.Glass, M.Y.Siddiqi and G.J.D.Kirk (2000) Comparative kinetic analysis of ammonium and nitrate acquisition by tropical lowland rice: implications for rice cultivation and yield potential. New Phytol. 145: 471-476.

Lejay,L., P.Tillard, M.Lepetit, F.D.Olive, S.Filleur, F.Daniel-Vedele and A.Gojon (1999) Molecular and functional regulation of two $\mathrm{NO}_{3}^{-}$uptake systems by $\mathrm{N}$ - and $\mathrm{C}$-status of Arabidopsis 
plants. Plant J. 18: 509-519.

Lin, C.-M., S.Koh, G. Stacey, S.-M.Yu, T.-Y.Lin and Y.-F.Tsay (2000) Cloning and functional Characterization of a constitutively expressed nitrate transporter gene, OsNRT1, from rice. Plant Physiol. 122: 379-388.

McElroy,D., M.Rothenberg and R.Wu (1990) Structural characterization of a rice actin gene. Plant Mol. Biol. 14: 163-171.

McGuffin,L.J., K.Bryson and D.T.Jones (2000) The PSIPRED protein structure prediction server. Bioinformatics 16: 404-405.

Okamoto,M., J.J.Vidmar and A.D.M.Glass (2003) Regulation of NRT1 and NRT2 gene families of Arabidopsis thaliana: responses to nitrate provision. Plant Cell Physiol. 44: 304-317.

Orsel,M., S.Filleur, V.Fraisier and F.Daniel-Vedele (2002a) Nitrate transport in plants: which gene and which control? J. Exp. Bot. 53: 825-833.

Orsel,M., A.Krapp and F.Daniel-Vedele (2002b) Analysis of the NRT2 nitrate transporter family in Arabidopsis. Structure and gene expression. Plant Physiol. 129: 886-896.

Page,R.D.M. (1996) TREEVIEW: An application to display phylogenetic trees on personal computers. Comput. Appl. Biosci. 12: 357-358.

Quesada,A., A.Galván and E.Fernández (1994) Identification of nitrate transporter genes in Chlamydomonas reinhardtii. Plant J. 5: 407-419.

Quesada,A., A.Krapp, L.J.Trueman, F.Daniel-Vedele, E.Fernández, B.G.Forde and M.Caboche (1997) PCR-identification of a Nicotiana plumbaginifolia cDNA homologous to the highaffinity nitrate transporters of the $c r n A$ family. Plant Mol. Biol. 34: 265-274.

Sasakawa,H. and Y.Yamamoto (1978) Comparison of the uptake of nitrate and ammonium by rice seedlings. Plant Physiol. 62: 665-669.

Sasaki,T., T.Matsumoto, K. Yamamoto, K. Sakata, T.Baba et al. (2002) The genome sequence and structure of rice chromosome 1. Nature 420: 312-316.

Sasaki,T., T.Matsumoto, B.A.Antonio and Y.Nagamura (2005) From mapping to sequencing, post-sequencing and beyond. Plant Cell Physiol. 46: 3-13.

Sonoda,Y., A.Ikeda, S.Saiki, T.Yamaya and J.Yamaguchi (2003) Feedback regulation of the ammonium transporter gene family AMT1 by glutamine in rice. Plant Cell Physiol. 44: 1396-1402.

Ta,T.C. and K.Ohira (1981) Effects of various environmental and medium conditions on the response of indica and japonica rice plants to ammonium and nitrate nitrogen. Soil Sci. Plant Nutr. 27: $347-355$.

Tateno, Y., N.Saitou, K.Okubo, H.Sugawara and T.Gojobori (2005) DDBJ in collaboration with mass-sequencing teams on annotation. Nucleic Acids Res. 33: D25-D28.

Thompson,J.D., D.G.Higgins and T.J.Gibson (1994) CLUSTAL W: improving the sensitivity of progressive multiple sequence alignment through sequence weighting, position-specific gap penalties and weight matrix choice. Nucleic Acids Res. 22: $4673-4680$.
Tong, Y., J.-J.Zhou, Z.Li and A.J.Miller (2005) A two-component high-affinity nitrate uptake system in barley. Plant J. 41: 442450

Trueman,L.J., A.Richardson and B.G.Forde (1996) Molecular cloning of higher plant homologues of the high-affinity nitrate transporters of Chlamydomonas reinhardtii and Aspergillus nidulans. Gene 175: 223-231.

Tusnáy,G.E. and I.Simon (1998) Principles governing amino acid composition of integral membrane proteins: applications to topology prediction. J. Mol. Biol. 283: 489-506.

Tusnáy, G.E. and I.Simon (2001) The HMMTOP transmembrane topology prediction server. Bioinformatics 17: 849-850.

Tsay,Y.-F., J.I.Schroeder, K.A.Feldmann and N.M.Crawford (1993) The herbicide sensitivity gene $\mathrm{CHL} 1$ of Arabidopsis encodes a nitrate-inducible nitrate transporter. Cell 72: 705-713.

Vidmar,J.J., D.Zhuo, M.Y.Siddiqi and A.D.M.Glass (2000a) Isolation and characterization of HvNRT2.3 and HvNRT2.4, cDNAs encoding high-affinity nitrate transporters from roots of barley. Plant Physiol. 122: 783-792.

Vidmar,J.J., D.Zhuo, M.Y.Siddiqi, J.K.Schjoerring, B.Touraine and A.D.M.Glass (2000b) Regulation of high-affinity nitrate transporter genes and high-affinity nitrate influx by nitrogen pools in roots of barley. Plant Physiol. 123: 307-318.

Wang, R. and N.M.Crawford (1996) Genetic identification of a gene involved in constitutive, high-affinity nitrate transport in higher plants. Proc. Natl. Acad. Sci. USA 93: 9297-9301.

Wang, R., R.Tischner, R.A.Gutiérrex, M.Hoffman, X.Xing, M.Chen, G.Coruzzi and N.M.Crawford (2004) Genomic analysis of the nitrate response using a nitrate reductase-null mutant of Arabidopsis. Plant Physiol. 136: 2512-2522.

Ware,D., P.Jaiswal, J.Ni, X.Pan, K.Chang, K.Clark, L.Teytelman, S.Schmidt, W.Zhao, S.Cartinhour, S.McCouch and L. Stein (2002) Gramene: a resource for comparative grass genomics. Nucleic Acids Res. 30: 103-105.

Youngdahl,L.J., R.Pacheco, J.J.Street and P.L.G.Vlek (1982) The kinetics of ammonium and nitrate uptake by young rice plants. Plant Soil 69: 225-232.

Yuan, Q., S.Ouyang, A.Wang, W.Zhu, R.Maiti, H.Lin, J.Hamilton, B.Haas, R. Sultana, F. Cheung, J. Wortman and C.R. Buell (2005) The institute for genomic research Osal rice genome annotation database. Plant Physiol. 138: 18-26.

Zhu, Q., M.I.Ordiz, T.Dabi, R.N.Beachy and C.Lamb (2002) Rice TATA binding protein interacts functionally with transcription factor IIB and RF2a bZIP transcriptional activator in an enhanced plant in vitro transcription system. Plant Cell 14: 795803

Zhou,J.-J., E.Fernández, A.Galván and A.J.Miller (2000) A high affinity nitrate transport system from Chlamydomonas requires two gene products. FEBS Lett. 466: 225-227.

Zhuo,D., M.Okamoto, J.J.Vidmar and A.D.M.Glass (1999) Regulation of putative high-affinity nitrate transporter $(N r t 2 ; 1 A t)$ in roots of Arabidopsis thaliana. Plant J. 17: 563-568. 